\title{
EL MAPA SINTÉTICO DE DESARROLLO TERRITORIAL: HERRAMIENTA WEB GEOGRÁFICA PARA EL APOYO EN LA TOMA DE DECISIONES TERRITORIALES
}

\author{
R. Martínez Cebolla and F. López Martín \\ Centro de Información Territorial de Aragón (CINTA). Departamento de Política \\ Territorial e Interior. Gobierno de Aragón, Edificio Pignatelli Paseo María Agustín, 36, \\ Puerta 14, $3^{a}$ planta 50.071 Zaragoza (España) \\ cinta@aragon.es

\section{Portolés Rodríguez} \\ Idearium Consultores S.L., Av. San Juan de la Peña 1, 50015 Zaragoza (España) \\ informacion@idearium-consultores.com
}

\begin{abstract}
Resumen: Desde el Centro de Información Territorial de Aragón (CINTA) se tiene la premisa inequívoca de ayudar de forma práctica a la Dirección General de Ordenación del Territorio del Gobierno de Aragón en el apoyo a la toma de decisiones territoriales. Su esfuerzo cognitivo, gracias al avance en las Tecnologías de la Información Geográfica (TIG), se ha centrado en desarrollar una aplicación web que refleje el diagnóstico territorial aragonés de forma sintética. El presente artículo versa sobre el Mapa Sintético de Desarrollo Territorial (MSD) como una decidida apuesta tecnológica del CINTA para reflejar de forma gráfica el estado del territorio aragonés y cómo pueden afectar, de forma positiva o negativa, las actuaciones que se hagan sobre el mismo en función de los componentes relacionales que se han de tener en cuenta cuando se quiere ordenar el territorio.
\end{abstract}

Palabras clave: indicador sintético, modelo territorial, ordenación del territorio, planificación territorial, estrategia territorial, sistema de información geográfica.

Abstract: CINTA works on the principle of offering assistance to the Spatial Planning Agency of the Government of Aragon in supporting territorial decision making. Its cognitive effort, thanks to advances in Geogra-

Recibido: 08-01-14. Aceptado: 02-04-14. 
phic Information Technologies, has focused on developing a web application that reflects synthetically a territorial diagnosis of Aragón. This paper deals with Synthetic Map of Territorial Development as a useful aid to reflect the status of aragonese territory in a visual way, and how possible interventions on territory could affect, in a positive or negative way, depending on relational elements involved on spatial planning.

Keywords: synthetic indicator, territorial model, management of the territory, territorial planning, territorial development, gis information system.

\section{Introducción}

\subsection{Motivación del proyecto}

La ordenación del territorio, entendida como la expresión espacial de la política económica, social, cultural y ecológica de toda sociedad, con multitud de objetivos, entre ellos el desarrollo socioeconómico y equilibrado de las regiones, la mejora de la calidad de vida, la gestión responsable de los recursos naturales, la protección del medio ambiente, y por último, la utilización racional del territorio (Carta Europea de Ordenación del Territorio, 1983) es a la vez una disciplina científica que aúna en un mismo prisma una técnica administrativa y una política comprendida como un enfoque interdisciplinario y global, cuyo objetivo es un desarrollo equilibrado de las regiones y la organización física del espacio según una directriz o estrategia rectora.

Aragón se encuentra en una encrucijada dentro del paradigma de la ordenación del territorio, desde su propio nacimiento como Comunidad Autónoma de España, en el marco del estado democrático en el que vive la sociedad española.

Los indicadores territoriales de Aragón siguen reflejando, tras más de tres décadas de Democracia, unos valores similares o quizá acrecentados de desequilibrio territorial. Pese a los esfuerzos unívocos de las fuerzas políticas en vertebrar el territorio, mediante la aprobación de figuras jurídicas ${ }^{1}$, la creación y delimitación de comarcas políticas-administrativas ${ }^{2}$ o proyectos de interés general autonómico (Plataformas logísticas, Walqa, Dinópolis, Motorland, etc.), con el objeto de generar motores de desarrollos alternos a la fuerza centrípeta que genera la macrocefalia de Zaragoza o fuerzas centrípetas exógenas al territorio aragonés, la realidad territorial es que no se está evitando

1. Ley 4/2009, de 22 de junio, de Ordenación del Territorio de Aragón, 2009.

2. Decreto Legislativo 1/2006, de 27 de diciembre, del Gobierno de Aragón, por el que se aprueba el texto refundido de la Ley de Comarcalización de Aragón. 
que el éxodo rural (Slomp, 2004) y la fuga de cerebros (Sorabji, 2005) se siga produciendo a otras regiones nacionales y, sobre todo, internacionales, acrecentado en los últimos tiempos dada la crisis económico-financiera existente.

Abordar y hacer una proyección del Modelo Territorial de una región es una labor compleja, más aún si cabe dentro del marco de incertidumbre que existe en la realidad económica española y europea. La coyuntura actual es que las relaciones exógenas en el Modelo Territorial aragonés tienen una potencia o peso superior a la que ya se tenía cuando se trazaron las Directrices Generales de Ordenación del Territorio. Este factor, motivado por la realidad territorial en la que se vive donde la mundialización de la economía financiera y las crisis económicas-especulativas están a la orden del día ${ }^{3}$, provoca que los procesos de especulación hayan desplazado totalmente a la economía productiva como fuente principal de acumulación de capital en las economías desarrolladas (Serrano Rodríguez, A., 2012).

Trazar el Modelo Territorial, entendido como el reflejo espacial de las formas en que la sociedad usa, organiza, se relaciona y transforma el territorio (Serrano, 1999), y llevar a cabo el seguimiento pormenorizado del mismo se tiene que convertir en el objetivo operativo dentro de la nueva Estrategia de Ordenación del Territorio de Aragón (EOTA) que está desarrollando el ejecutivo aragonés ${ }^{4}$.

Este nuevo Modelo Territorial debe contestar tres preguntas claves. La primera, es necesario conocer de qué situación territorial se viene. Este hecho implica estudiar y analizar el recorrido y el grado de ejecución de las Directrices Generales y Parciales (Pirineos y Matarraña/Matarranya) de Ordenación del Territorio aplicadas en la Comunidad Autónoma de Aragón en las últimas dos décadas, así como estudiar y comprender de qué forma (positiva, neutra o negativa) ha influido el desarrollo de las comarcas dentro del territorio aragonés.

La segunda, es conocer en qué situación estamos. Resolver esta cuestión implica conocer la foto oficial, es decir, disponer de la radiografía desde el punto de vista territorial para tener en cuenta tanto las fortalezas y oportunidades como las debilidades y sobre todo amenazas que posee la región. Aragón, cuenta hoy, pese a los esfuerzos en la mejora del sistema de transportes y la creación de proyectos de interés general dentro del sector secundario y terciario, con un desequilibrio demográfico mayor que el existente hace medio siglo donde el éxodo rural no ha sido reparado sino acrecen-

3. Se recomienda al lector la lectura integra del interesante prefacio de José Manuel Barroso en la comunicación elaborada, en el año 2010, por la Comisión Europea sobre Europa 2020. Una estrategia para un crecimiento inteligente, sostenible e integrador.

4. Acceso a la dirección web oficial, del Gobierno de Aragón, donde se publica la información más reciente sobre la Estrategia de Ordenación Territorial de Aragón (EOTA): http://www.aragon.es/DepartamentosOrganismosPublicos/Departamentos/PoliticaTerritorialInterior/AreasTematicas/OrdenacionTerritorial/EOTA. 
tado, el tejido empresarial está diezmado por la realidad de la crisis económica global y la pérdida no tanto de calidad de vida sino del grado de aprecio que implica que el ciudadano se arraigue y haga prosperar el territorio de una forma sostenible.

El concepto de "ciudad menguante" (Sánchez, 2009) es, por lo tanto, aplicable a escala regional al territorio aragonés si no se llevan a cabo actuaciones claves de diversificación funcional en los diferentes componentes que entran en juego dentro de la ordenación territorial. A este respecto, las relaciones tanto directas como indirectas entre los componentes territoriales - población, economía, medio ambiente- son determinantes para generar un Modelo Territorial que se ajuste a la realidad global en la que está inmerso Aragón.

El éxito del nuevo Modelo Territorial tiene que conseguir contrarrestar el peso de inercias heredadas (políticas, económicas, sociales y culturales) que influyen a la actual situación dificultando su adaptación al nuevo contexto extra-regional bajo el concepto de "path dependency" (Martin y Sunley, 2006 y Bontje, 2011 y Röhring y Gailing, 2011) $)^{5}$. A este respecto, el Modelo Territorial, tal como se refleja en la Figura № 1, debe tener localizados de forma clara la conjunción de los diferentes componentes relacionados con la existencia de recursos (potenciales), actores (sociedad, sector público y privado, comunidad científica) y valores (culturales y naturales esencialmente) existentes dentro del territorio aragonés (Mendez, 2010). Conocer las entradas y salidas del sistema implica el conocer qué recursos específicos, de capital no estrictamente económico, sino también humano, son capaces de ser movilizados y puestos en valor para el conjunto de actores públicos y privados con el objetivo de provocar la mayor cantidad de sinergias. Estas sinergias son las que permitirán la constitución de redes de cooperación, tanto formal como informal, que implican intercambio de información y conocimiento y desarrollo. En suma, de nuevos productos y/o mercados específicos de oferta y demanda no sólo para la sociedad aragonesa sino por la sociedad global.

La tercera cuestión es, qué Modelo Territorial se quiere. Una vez conocida la radiografía, se tiene que plasmar de forma literal y gráfica cuál es la estrategia y los objetivos operativos para combatir el fenómeno de "resiliencia urbana" (Polèse, 2010) aplicado a la óptica territorial. Es decir, el Modelo Territorial se ha de enfrentar a los procesos de declive

5. Path dependency pueden entenderse como el uso continuo de un producto o práctica basada en la preferencia histórica. La "dependencia de la ruta" se produce porque a menudo es más fácil o más rentable continuar a lo largo de un camino ya establecido que para crear una nueva ruta, es decir, la dependencia de ruta sería una región que está desarrollada dependiendo de un único tipo de sector productivo como, por ejemplo, es el caso de Detroit con la industria del automóvil que tras la crisis del fordismo provocó un impacto territorial negativo con indudables consecuencias económicas, sociales y ambientales. Se recomienda al lector la lectura del interesante artículo del Jot Down Cultural Magazine (Rodríguez, 2013). Detroit: así se hundió el Titanic del capitalismo estadounidense. Acceso a la dirección web oficial: http://www.jotdown.es/ 2013/02/detroit-asi-se-hundio-el-titanic-del-capitalismo-estadounidense. 
y revertirlos lo que implica un aumento de las potencialidades y ventajas competitivas pero también supone un incremento, por un lado, de la cohesión social, como, por otro lado, de la calidad de vida y la sostenibilidad económica y ambiental del territorio.

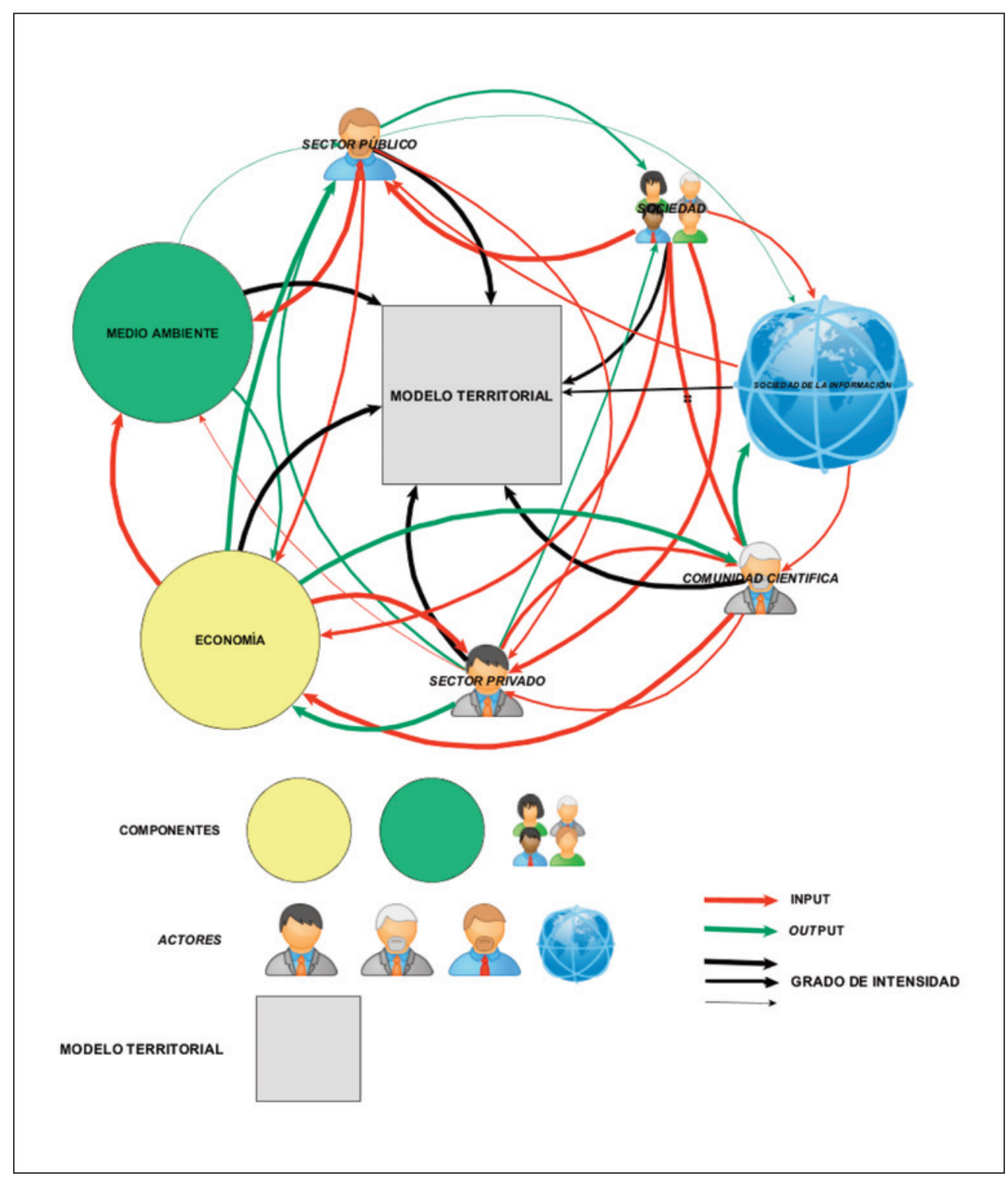

Figura 1. Diagrama de flujos entre los actores que intervienen dentro del Modelo Territorial. 
En suma, esta última pregunta ha de contestar el qué quiere ser el Gobierno de Aragón como realidad territorial a corto y medio plazo, es decir, un territorio latente, con claros síntomas de crisis de carácter "fordista", y acompasado al devenir nacional y europeo que se está viviendo actualmente, -crisis del Euro- o, por contra, un territorio emergente que apueste por una nueva realidad territorial basada en el consenso de los diferentes actores que conviven y se relacionan en el mismo territorio. Una nueva realidad territorial que pueda reorganizar sus recursos económicos, humanos y ambientales en virtud de la nueva curva de oferta y demanda generada en la presente "sociedad de consumo 2.0" donde el concepto de producto (Levitt, 1981) ha sido reemplazado por el concepto de "servicio global".

\subsection{La decisión de dar el paso}

Ante este cúmulo de preguntas, que han de ser justificadas y planteadas en un necesario documento de estrategia territorial, el Centro de Información Territorial de Aragón (CINTA) no quiere permanecer como un espectador más. Es por ello que quiere tomar parte activa ayudando a la toma de decisiones para que los órganos directivos y consultivos tengan más argumentos para contestar a las preguntas anteriormente comentadas.

El CINTA nace, hace ya más de una década, tanto como servicio de apoyo a las diferentes administraciones públicas como directamente a los ciudadanos, en todo aquello relacionado con la información y documentación sobre ordenación del territorio aragonés.

Desde su creación, ha desarrollado una extensa labor de recopilación, almacenamiento y, finalmente, publicación de la información territorial a través de la plataforma electrónica del Sistema de Información Territorial de Aragón (SITAR) ${ }^{6}$. No obstante, la realidad territorial no es ajena a este Centro, que no sólo quiere ser una unidad administrativa que gestione la Información Geográfica (IG) del territorio aragonés, sino que quiere ser un actor que se implique y ayude en la toma de decisiones territoriales a los órganos consultivos en materia de ordenación del territorio.

Su esfuerzo cognitivo, gracias al avance en las Tecnologías de la Información Geográfica (TIG), le ha permitido desarrollar una aplicación web cuyo objetivo es reflejar el diagnóstico territorial aragonés de forma sintética. Esta herramienta es el Mapa Sintético de Desarrollo Territorial, definida como la aplicación web que representa el estado actual del desarrollo territorial de las comarcas de Aragón basándose en un indicador sintético que aúna la conjunción de los componentes que intervienen en el territorio.

6. URL de acceso al Sistema de Información Territorial de Aragón: http://sitar.aragon.es 
Los responsables políticos y técnicos pueden utilizar el software de forma práctica pudiendo discernir qué indicadores estadísticos se quieren potenciar sobre otros dentro de cada componente y, a su vez, qué actuaciones se quieren llevar a cabo para generar la sinergia territorial necesaria para mejorar de forma sostenible el territorio.

\section{Desarrollo del Mapa Sintético de Desarrollo Territorial}

\subsection{Objetivo de la herramienta}

El objetivo inicial que se marcó el CINTA a la hora de hacer realidad esta herramienta es saber si era posible hacer converger el conjunto de componentes que intervienen de una forma u otra en el territorio y poder representarlos de forma gráfica dentro de una aplicación Web cartográfica.

De este modo, la fase inicial del presente proyecto consistió en dotar a la Dirección General de Ordenación del Territorio del Gobierno de Aragón de una herramienta capaz de realizar una síntesis real del desarrollo territorial existente dentro de la Comunidad Autónoma. Para ello se tomó la comarca ${ }^{7}$, como unidad mínima de información, con el objetivo de representar gráficamente el desarrollo territorial mediante un indicador sintético que representa la interrelación de los siguientes componentes: población, economía, territorio y accesibilidad.

El resultado es una aplicación Web que sirve como cuadro de mando. Representa un mapa de coropletas reflejando sintéticamente la realidad territorial aragonesa permitiendo traducir lo que representa un cambio o un conjunto de actuaciones de incidencia territorial dentro del conjunto de comarcas aragonesas.

Como herramienta se corresponde con otras iniciativas análogas realizadas por otros organismos o entidades que han apostado por la generación de cuadros de mandos que permiten definir las estrategias a nivel territorial o urbanístico (Unión Europea. Inspire policy making by territorial evidence. ESPON HyperAtlas o Research Institute for Knowledge Systems. Metronamica o Ebrópolis. Marco estratégico Zaragoza 2020) partiendo de una batería de indicadores que reflejen la realidad del ámbito territorial que es objeto del estudio.

En suma, la herramienta quiere apoyar a las políticas en materia de ordenación del territorio de manera que; uno, permita una exploración del diagnóstico actual, así como del conjunto de proyecciones distintas al permitir el juego tanto con los componentes

7. La Comunidad Autónoma de Aragón está compuesta de 32 Comarcas más la Delimitación Comarcal de Zaragoza. 
que afectan al territorio como de las actuaciones que se puede realizar sobre el mismo y, dos, permita evaluar el impacto de políticas alternativas al poder compararlo con el estado inicial.

De este modo, ambos tipos de análisis pueden ser incorporados en un proceso participativo en el que están representados los diferentes grupos de componentes y/o actores. La herramienta permite dar soporte para facilitar ese proceso de toma de decisiones de manera que, se pueden crear estadios evolutivos para poder evaluar y visualizar los impactos de una combinación determinada de componentes dentro del territorio.

\subsection{El presente de la herramienta}

El Mapa Sintético de Desarrollo Territorial cuya dirección de acceso web es: http://sitar.aragon.es $/ \mathrm{MSD}^{8}$ se crea con la exclusiva vocación de ser, una herramienta de apoyo a la estrategia territorial del Gobierno de Aragón en la toma de decisiones relacionadas con el territorio aragonés. Las características básicas de la aplicación web son las siguientes:

- Es conforme al entorno tecnológico existente en SITAR.

- Su desarrollo está basado en productos de software libre (JQUERY, OpenLayers).

- Tiene una independencia total de productos software concretos y licencias comerciales.

- Reutiliza servicios web existentes en SITAR (estándar WMS del OGC).

- Está basado en paradigma IDE (Infraestructuras de Datos Espaciales).

La justificación de la elección de esta solución tecnológica está directamente relacionada con dos factores. De una mano, la obligación de cumplir con las especificaciones que Aragonesa de Servicios Telemáticos ${ }^{9}$ impone en la creación de aplicaciones dentro de la plataforma tecnológica disponible. De otra mano, la necesidad de cumplir con la Directiva INSPIRE, a la hora de crear servicios que puedan ser interrogados por otros entornos tecnológicos sin tener la obligación de acceder directamente a la aplicación nativa, facilitando el grado de interoperabilidad tanto de la información como del servicio Web (WMS) ofrecido por la presente aplicación.

8. La presente aplicación se publicará oficialmente en la dirección Web indicada el último trimestre del año 2014.

9. Aragonesa de Servicios Telemáticos (AST) es la Entidad Pública del Gobierno de Aragón encargada de la atención a usuarios, consultoría y gestión de proyectos TIC y telecomunicaciones. 
La siguiente Figura № 2 representa la arquitectura de despliegue de la presente aplicación:

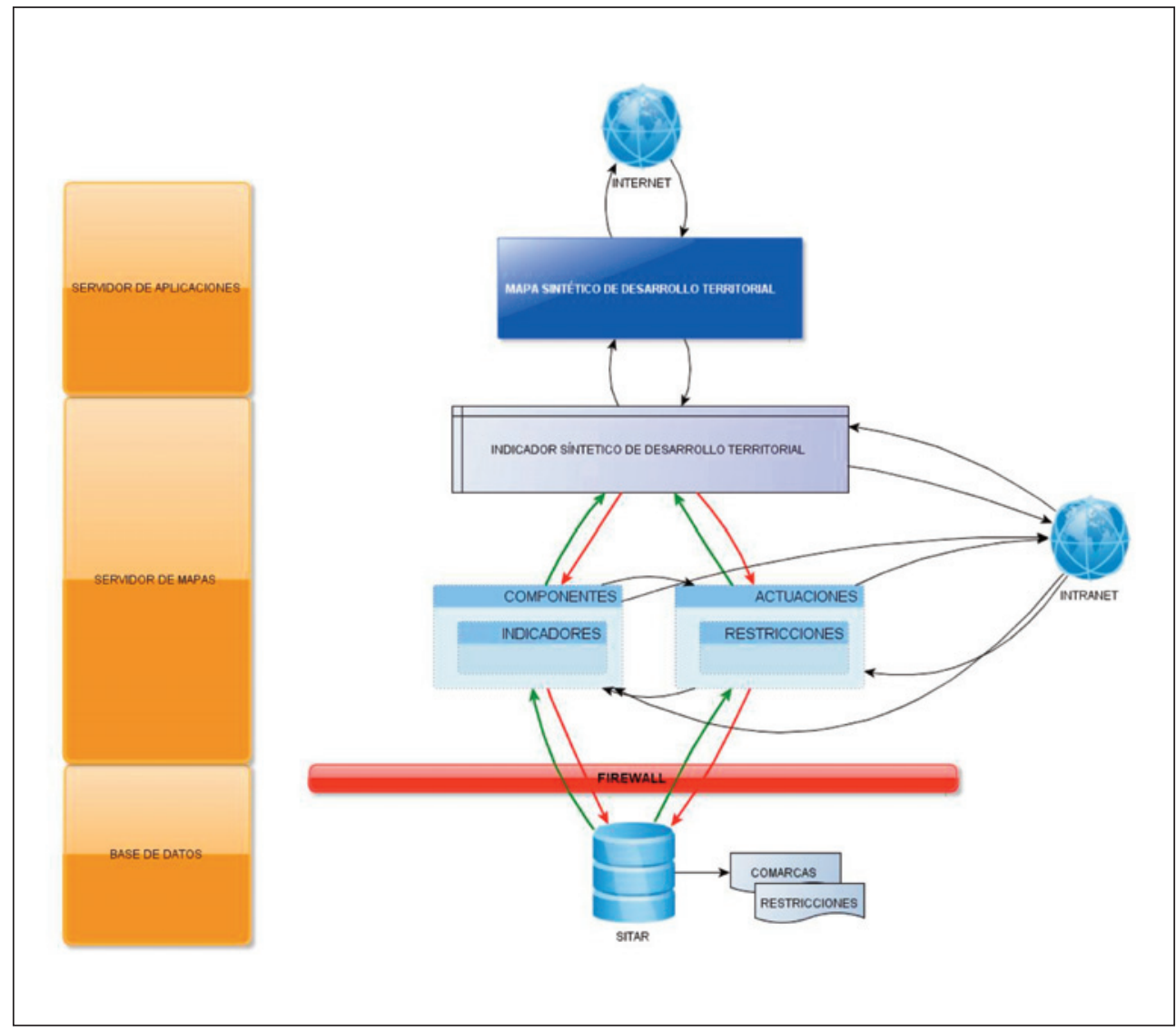

Figura 2. Arquitectura de despliegue del Mapa Sintético de Desarrollo Territorial.

La aplicación web cartográfica trabaja con variables de tipo estadístico englobados con el objetivo de definir el Indicador Sintético de Desarrollo Territorial. Esta herramienta es escalable al número de indicadores estadísticos que se quieran incorporar, con el objetivo de actualizar el indicador sintético teniendo en cuenta la comarca como unidad mínima de trabajo.

La herramienta permite calcular el estado de las distintas comarcas aragonesas a nivel global (para todo el conjunto de comarcas) o específico (para una comarca determinada) en base a los componentes citados anteriormente. Actualmente, Figura № 3, 
la aplicación trabaja con esos cuatro componentes citados que agrupan, hasta la fecha, 16 indicadores estadísticos con componente territorial ${ }^{10}$.

Además, está preparada para el manejo de otros indicadores estadísticos, que por el momento no intervienen en el cálculo y otros indicadores con componente territorial que se estimen necesario introducir a corto o medio plazo.
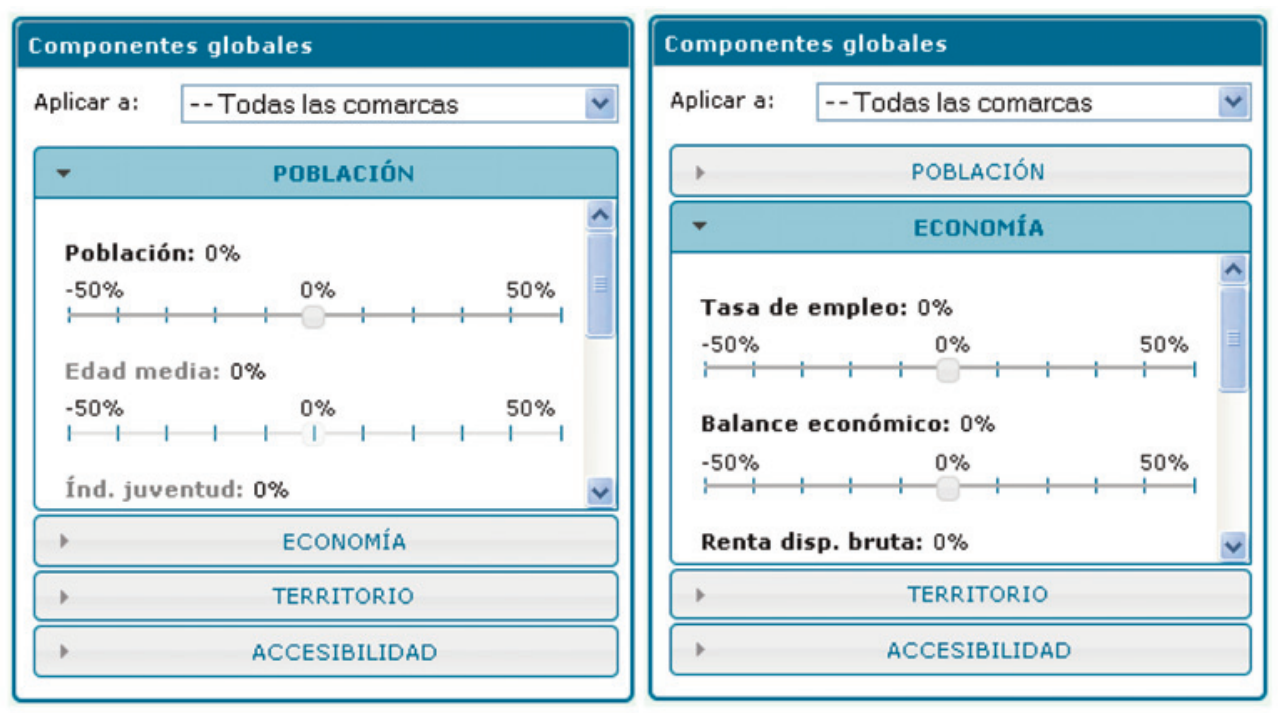

Figura 3. Ejemplos de la ventana de componentes globales del Mapa Sintético de Desarrollo Territorial.

El índice de estado de las comarcas se calcula como el sumatorio de los valores que toman dichas variables en cada comarca, dividido por el número de indicadores que entran en juego. El índice se clasifica en seis estados posibles en los que se clasifica el Índice Sintético de Desarrollo Territorial. Los estados, tal como refleja la Figura № 4, son los siguientes: Deprimido, Negativo clase 1 y 2, Neutro, Positivo clase 1 y 2.

10. Los indicadores, agrupados por componente, son los siguientes: Componente población (Datos procedentes del Instituto Aragonés de Estadística (IAEST. 2012): Datos de población (cantidad y densidad de población), tasa de emigración, índices de juventud y envejecimiento. Componente economía (Datos procedentes del IAEST. 2012): Tasa de empleo, balance económico, renta disponible bruta, inversión y gasto. Componente territorio (Datos procedentes del IAEST. 2012): Renta bruta per cápita, disponibilidad de agua y limitaciones ambientales. Componente accesibilidad (Datos procedentes del Departamento de Educación, Universidad, Cultura y Deporte, IAEST e Instituto Aragonés de Fomento, IAF. 2012): Equipamiento educativo, sanitario e industrial. 




Figura 4. Leyenda y método de clasificación de los valores del Indicador Sintético de Desarrollo Territorial.

El nombre dado a los tipos de estado implica el grado de desarrollo territorial que posee una determinada Comarca respecto al resto de comarcas del territorio aragonés.

Estos estados siempre estarán representados dentro del Mapa Sintético, es decir, el método de clasificación utilizado dentro de la aplicación siempre "romperá" la clasificación de valores en seis estados. Por lo que, al menos una comarca siempre estará representada en un estado por lo que cualquier acción que el usuario realice tanto en la ventana de componentes globales como en el de actuaciones tendrá fiel reflejo en el resultado final del mapa. La relación entre las comarcas es, por lo tanto, global por lo que las afecciones siempre afectan al resultado final del mapa sintético. El método de clasificación activo por defecto es por rotura natural basado en el método de Jenks ${ }^{11}$.

A partir del estado de partida de las comarcas, que se origina tras el estudio de las variables estadísticas que posee actualmente cada comarca, la herramienta permite comprobar cómo variarían los estados correspondientes a las comarcas, en función de diferentes escenarios posibles. Estos escenarios se pueden establecer mediante la mejora o empeoramiento de las variables (Figura № 3) o mediante actuaciones concretas (Figura № 6). Las herramientas de la ventana "Componentes globales" de la presente aplicación permiten modificar los valores de los indicadores hasta un 50\%, tanto en positivo (acción de mejora de la variable) como en negativo (acción de empeoramiento de la variable). Estos cambios se pueden aplicar al conjunto de las comarcas y/o de forma específica a cada comarca.

11. El método Jenks, genera intervalos (rangos) dentro de series numéricas siendo útil para la aplicación típica para rangos de valores en las leyendas de los mapas y sobre todo, en el caso del territorio aragonés, para constatar la diferencia de la vertebración territorial entre las comarcas existentes. La aplicación de este algoritmo dentro del mapa sintético es de gran utilidad dado que procede comparando de forma iterativa las sumas de las diferencias al cuadrado entre valores observados dentro de cada clase y las medias de las clases, esto es, que la mejor clasificación se considera cuando se encuentran aquellos umbrales que minimizan la suma intra-clase de diferencias al cuadrado (Jenks, 1967). 
No obstante, tal como se observa en la Figura № 5, la aplicación permite generar el mapa de coropletas basándose en los siguientes métodos de clasificación de los valores obtenidos para el indicador sintético. Estos métodos son: por cuantil y desviación estándar, aunque siempre cumpliendo con la premisa de que al menos una comarca esté encuadrada en alguno de los seis estados posibles de "desarrollo" territorial.

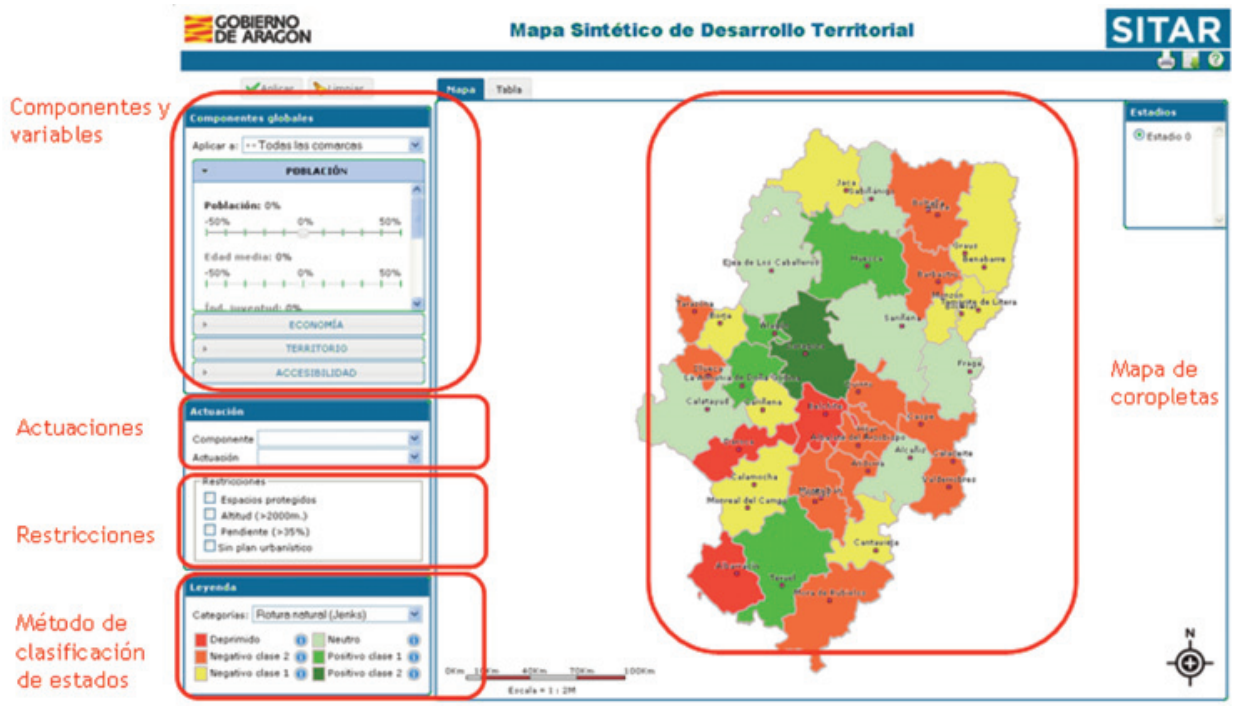

Figura 5. Vista gráfica de la aplicación Web: Mapa Sintético de Desarrollo Territorial.

Con las herramientas de la ventana “Actuación”, Figura № 6, se puede definir la creación, mejora o eliminación de actuaciones con incidencia territorial, como por ejemplo; polígonos industriales o carreteras, así como la ejecución de actuaciones de interés autonómico en cualquier punto de la geografía aragonesa ${ }^{12}$.

En el caso de que la actuación que se quiera reflejar sea una creación, se dibuja sobre el mapa el punto o polilínea que la representa geográficamente. En el caso de mejora o eliminación se seleccionará en el mapa el componente concreto sobre el que se desea actuar. Las actuaciones, Figura № 7, provocan la bonificación o penalización de determinadas variables en las comarcas en las que se ubiquen.

12. El número total de actuaciones que se pueden realizar hasta la fecha son ocho agrupadas en cuatro tipos: actuación de interés autonómico, carretera, polígono industrial y polígono industrial con ocupación. 




Figura 6. Ventana de actuaciones y aplicación de restricciones del Mapa Sintético de Desarrollo Territorial.

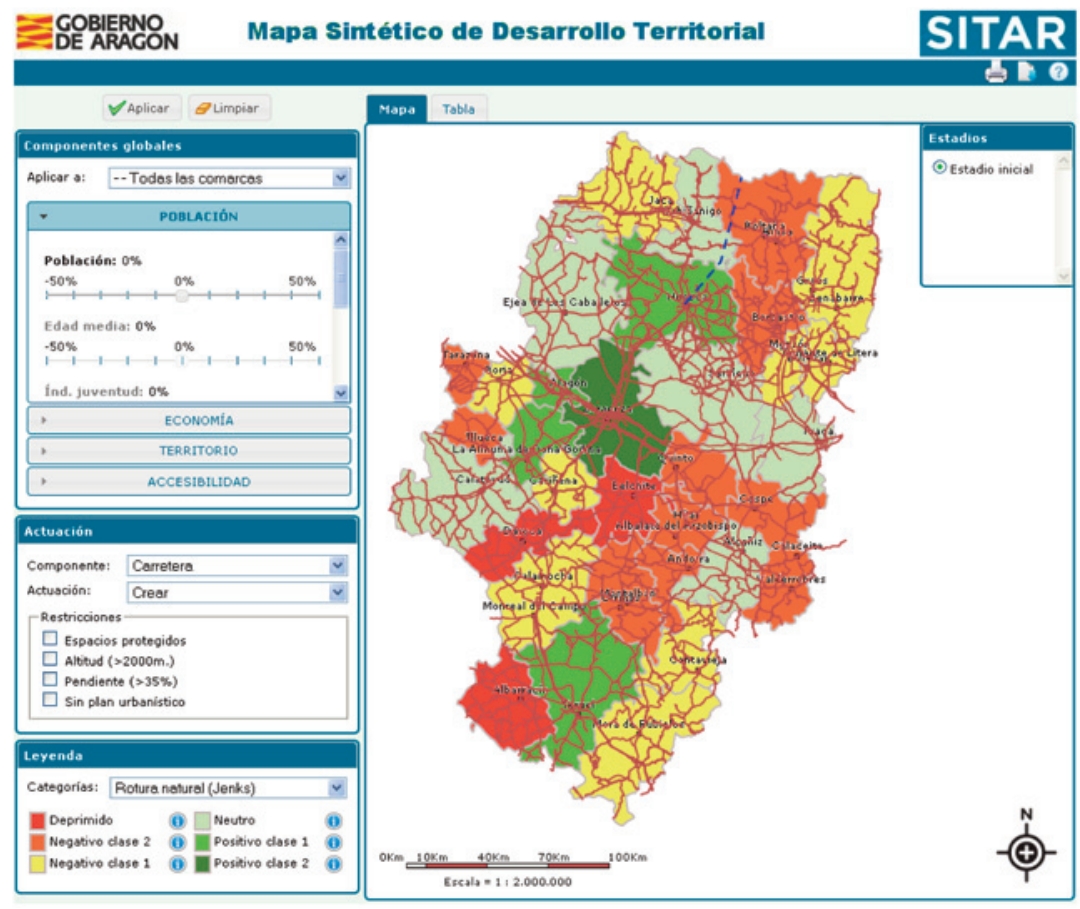

Figura 7. Creación de nueva actuación sobre el territorio (carretera) marcada en línea discontinua de color azul. 
De esta forma, el índice de estado (IE) de una comarca resultante de las modificaciones realizadas, se calcula mediante la siguiente fórmula:

$$
I E=\frac{\sum_{i=1}^{n}\left(v_{i}\left(1+c_{i}+\sum_{j=1}^{8} a_{i j}\right)\right)}{n}
$$

Fórmula 1. Fórmula de cálculo del indicador sintético de desarrollo territorial utilizado en la versión inicial de la aplicación cartográfica.

Donde:

- 1 es el valor del estado inicial.

- n es el número de variables.

- $v_{i}$ es el valor de la variable en la comarca.

- $c_{i}$ es la modificación de $v_{i}$ establecida para la comarca (de forma específica o para todas las comarcas).

- $a_{i j}$ es la bonificación correspondiente a la actuación de tipo j realizada en la comarca.

El resultado de esa fórmula es el valor final del indicador sintético de desarrollo territorial que la aplicación cartográfica dibuja mediante un mapa de coropletas. En determinadas circunstancias, algunas de las actuaciones de creación anteriores no siempre serán posibles de aplicar de forma directa, sino que requerirán un análisis de la viabilidad de las mismas. Esto es lo que se refleja en la ventana de restricciones, Figura № 8, la cual engloba las siguientes categorías: Espacios protegidos, Mapa de altitud con valor superior a 2.000 metros, Mapa de pendientes superiores al 35\% y Municipios sin Figura de Planeamiento Urbanístico. De este modo, el índice de estado de una comarca resultante de las modificaciones realizadas, se recalcula en función de la fórmula matemática anteriormente detallada.

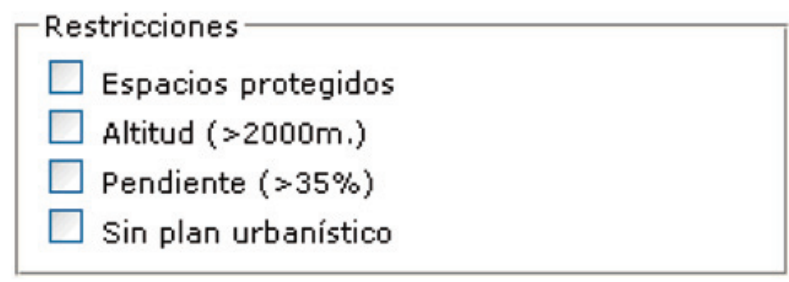

Figura 8. Cuadro de diálogo para la selección de restricciones. 
Las restricciones están por defecto desactivadas y es el usuario quien debe marcarlas, Figura № 9, para que interactúen con la lógica de la aplicación a la hora de calcular un nuevo estadio.

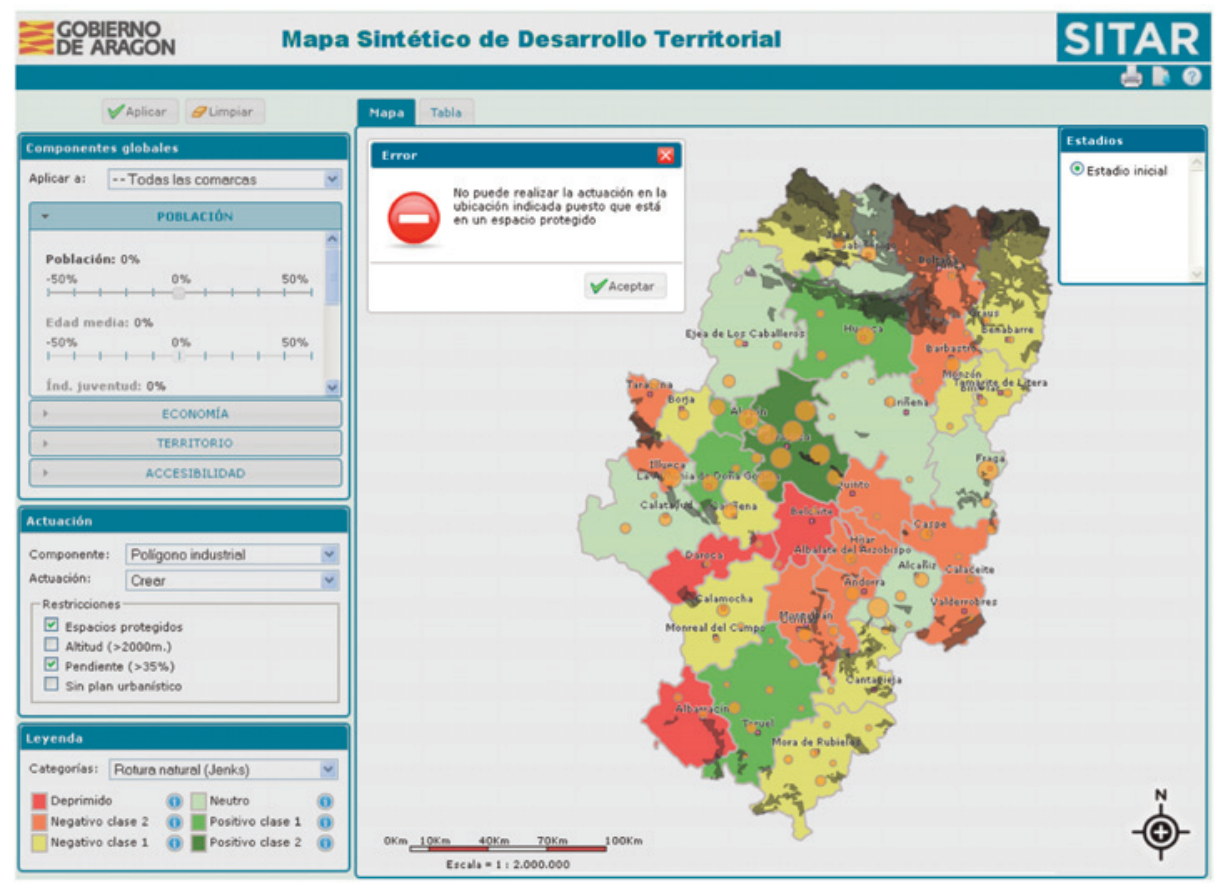

Figura 9. Mensaje de aviso de la aplicación al intentar crear un polígono industrial dentro de un lugar donde hay una restricción (existencia de espacio protegido en color negro).

Tanto los componentes como las actuaciones también son escalables o modificables como en el caso de los indicadores estadísticos que se relacionan en la ventana de componentes globales, es decir, la aplicación está preparada para que el órganos directivos puedan modificar la aplicación para que trabaje con nuevos tipos de indicadores así como tipos de actuaciones o restricciones existentes en el territorio. Este aspecto es muy relevante e innovador desde el punto de vista del CINTA dado que la ordenación del territorio es una disciplina a la que hay que prestar un seguimiento real y efectivo por lo que es necesario que los parámetros de esta aplicación puedan ser modificados ${ }^{13}$.

13. Es indudable que la creación de un polígono industrial, por ejemplo, no es por sí mismo un motor de desarrollo si en él no se implanta el tejido empresarial necesario para generar las sinergias socioeconómicas que este hecho conlleva. Para ello se necesita saber cómo se han desarrollado en el tiempo y qué impacto territorial han tenido este tipo de acciones sobre el territorio. 
Cada vez que se calcula un nuevo estadio, se recalcula la clasificación (estado) del mismo en base a los nuevos valores obtenidos. Con los nuevos valores y la nueva clasificación se procede a la actualización del mapa y la tabla mostrados por la aplicación, Figura № 10, manteniendo un histórico de los estadios anteriores para ver la evolución de los estadios, por los que van pasando las comarcas aragonesas, como resultado de las modificaciones planteadas al estadio inicial. De esta manera, se permite volver al estadio anterior para conocer en todo momento, qué implicaciones tienen las modificaciones sobre los componentes o las actuaciones realizadas sobre el territorio reflejado gráficamente a través del indicador sintético.

En suma, la presente aplicación cumple con las expectativas planteadas a inicio del proyecto, esto es, disponer de una herramienta capaz de "interrogar" a los componentes que afectan al territorio y extraer una conclusión sintética sobre el mismo.

\subsection{El futuro de la herramienta:}

La Dirección General de Ordenación del Territorio en vista del potencial de la presente herramienta, elaborada por el CINTA, quiere ahondar en la mejora de la misma con el objetivo de permitir una flexibilización de sus capacidades y ser referente como herramienta de toma de decisiones territoriales para los órganos consultivos en materia de Ordenación o Planificación Territorial.

Las acciones de mejora de la presente aplicación son numerosas y factibles una vez visto el potencial comentado en el anterior apartado. Estas acciones son de dos tipos: de contenido y de ergonomía.

Las primeras, en cuanto al contenido, implican una mejora de la lógica de negocio de la aplicación para poder responder de una forma más precisa a las modificaciones realizadas por el usuario. De entre ellas hay que destacar la posibilidad de incorporar distintas vistas gráficas del mapa sintético, como mínimo a escala municipal, permitiendo obtener una visualización más detallada del mapa correspondiente a Aragón -aunque la unidad mínima del resultado generado sea siempre la comarca- o generar relaciones de proximidad territorial, con diferentes tipos de influencias asociadas, entre los distintos actores implicados en los cálculos del sistema -actualmente sólo son las comarcas- agregando la relación de estrategias desarrolladas a nivel autonómico, estatal y europeo dado que se es consciente que Aragón per se, no es una isla o sistema territorial cerrado donde las acciones tomadas a nivel inter-autonómico o supra-autonómico no afectan a la región en sí.

Por otro lado, se estima conveniente, de una mano, establecer un procedimiento de actualización automático de las fuentes de datos utilizadas, especialmente para los casos de variables generadas periódicamente como por ejemplo la tasa de empleo, el IPC, 


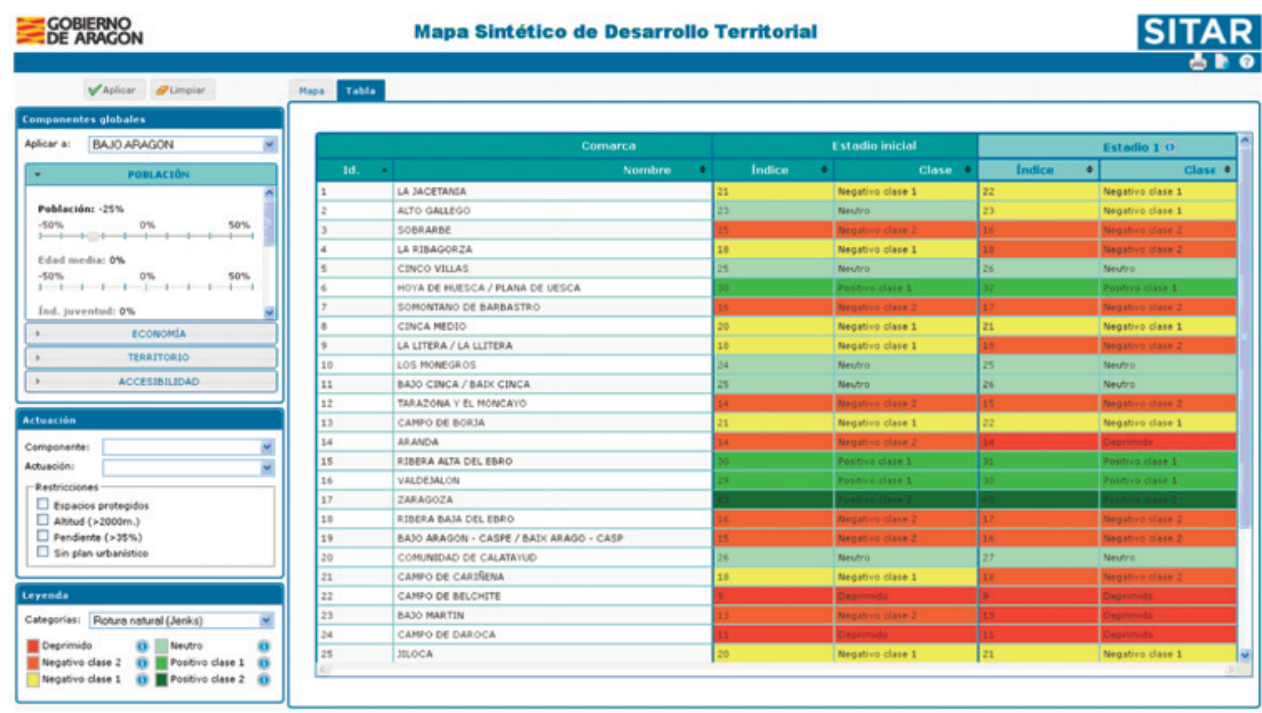

\section{GOBERNO}

SITAR


Figura 10. Resultados tras aplicar una modificación del componente población y documento de impresión con mapa e informe de resultados. 
etc. o cualquier indicador estadístico que se pueda utilizar en la presente aplicación que tenga una periodicidad inferior a la anual y, de otra mano, desarrollar un módulo de "autoaprendizaje" a través de aplicación de técnicas multicriterio para poder extraer tendencias y proyectar evolución prevista para las distintas variables incorporadas.

Las segundas, suponen una mejora de los aspectos ergonómicos de la aplicación para dar una mayor flexibilidad a la hora de obtener las diferentes posibilidades de resultados que se pueden rescatar de esta aplicación. De entre ellas hay que destacar la mejora del informe de actuaciones incluyendo información más detallada de las acciones realizadas por el usuario, generar nuevos diferentes tipos de clasificación de los resultados, habilitar la posibilidad de configurar que las restricciones se apliquen a sólo un subconjunto de actuaciones o, permitir el establecimiento de diferentes áreas de influencia de las actuaciones según el valor que tengan otras variables vinculadas.

Además, se estima de indudable interés, el permitir la posibilidad de mantenimiento de sesiones de trabajo, es decir, que la aplicación permitirá guardar el estado actual de las actuaciones realizadas y la carga posterior en la herramienta para continuar en el mismo punto en que se dejó en cualquier estación de trabajo en el que se quiera trabajar, así como incorporar a la herramienta la perspectiva evolutiva a lo largo del tiempo o superponer documentos de prospectiva territorial como apoyo a la toma de decisiones.

En definitiva, el recorrido previsto para la presente aplicación es largo si se atiende a lo descrito, más aún si se liga al desarrollo de dos proyectos que están planificados dentro del Plan Cartográfico de Aragón 2013-2016, que proveerán a la presente aplicación de los suficientes argumentos y contenidos para que el indicador sintético de desarrollo territorial sea más preciso. Estos proyectos son la redacción de los Documentos Informativos Territoriales, exigidos en la Ley de Ordenación del Territorio de Aragón (artículo 44, LOTA 2009) y el desarrollo del Sistema de Indicadores Territoriales de Aragón (SITA) que permita explotar el conjunto sistémico de indicadores con incidencia territorial. Ambos proyectos tendrán reflejo real a lo largo de 2014 y serán el semillero esencial de la presente aplicación cartográfica.

\section{Conclusiones}

Evaluar el impacto de políticas territoriales no es una tarea fácil, dado que implica tener una buena comprensión de varios dominios científicos, para poder equilibrar las ventajas y desventajas que implica realizar determinadas actuaciones sobre el territorio.

La presente aplicación sirve como ayuda en la toma de decisiones, mostrando el impacto que puede suponer la práctica territorial a través de una amplia gama de posibilidades (componentes, actuaciones y restricciones). Además, sirve para la discusión 
de todos los actores involucrados en el proceso de toma de decisiones y, sobre todo, y lo más importante, es que permite ensayar sobre el territorio antes de realizar grandes inversiones que pueden implicar cambios irreversibles sobre una región.

La finalidad de esta aplicación radica en la posibilidad de contestar a preguntas como: ¿Cuáles son las implicaciones espaciales del sobreenvejecimiento de la población?, ¿El desarrollo compacto del territorio proporcionará un futuro más sostenible que el crecimiento urbano disperso o viceversa?, ¿Cuál es el beneficio de la construcción de un aeropuerto en la atracción de la actividad secundaria o terciaria? o ¿Cuál es el impacto para el medio ambiente cuando se aplica una determinada actuación sobre el territorio?

El futuro y potencial de este tipo de herramienta es innegable. Con el Mapa Sintético de Desarrollo Territorial se aborda el estudio y análisis del impacto de las políticas territoriales a escala regional, pero es posible profundizar en la escala de estudio, es decir, a escala municipal o local. Para ello, es necesario establecer los cauces formales para conocer las tendencias y aplicaciones generadas a esas diferentes escalas, con el objetivo de mejorar bajo los principios de subsidiariedad e interoperabilidad.

El CINTA quiere erigirse en un centro referente de apoyo a los órganos encargados de la ordenación del territorio a nivel autonómico y su apuesta se concentrará en la mejora de esta aplicación con el objetivo de que sirva para definir un Modelo Territorial de Aragón acorde con la sociedad desarrollada en la que se enmarca, reflejando la gran cantidad de relaciones de entrada y salida que posee este tipo de sistemas donde cada acción sectorial que incide sobre el territorio, puede tener consecuencias sobre el desarrollo sostenible del mismo.

\section{Bibliografía}

Bontje, M.A., Musterd, S., y Pelzer, P. (2011) Inventive city-regions: Path dependence and creative knowledge strategies. Farnham, England; Burlington, Vt.: Farnham, England; Burlington, Vt.: Ashgate.

Comisión Europea. Estrategia Territorial Europea. Hacia un desarrollo equilibrado y sostenible del territorio de la UE (en línea): Unión Europea: http://ec.europa.eu/regional_policy/sources/docoffic/official/reports/pdf/sum_es.pdf (Consulta: 01.02.2012).

Comisión Europea. Europa 2020. Una estrategia para un crecimiento inteligente, sostenible e integrador (en línea): Disponible en: http://ec.europea.eu/commission_2010-2014/president/news/documents/pdf/20100303_1_es.pdf (Consulta: 01.02.2012).

Consejo de Europa. Carta Europea de Ordenación del Territorio (en línea): http://ec.europa.eu/regional_policy/sources/docoffic/official/reports/pdf/sum_es.pdf (Consulta: 01.02.2012).

De Miguel, R., Ezquiaga, J.M. (2012) Hacia una ordenación territorial metropolitana renovada en Europa: los planes de las regiones urbanas de París, Londres, Berlín y Roma. Ciudad y Territorio. Estudios Territoriales, 174, 669-688. 
Decreto 205/2008, de 21 de octubre, del Gobierno de Aragón, por el que se aprueban las Directrices Parciales de Ordenación Territorial de la Comarca del Matarraña/Matarranya. En Boletín Oficial de Aragón, no 184 (en línea): http://www.boa.aragon.es/cgi-bin/EBOA/BRSCGI?CMD=VEROBJ\&MLKOB=307745762323 (Consulta: 06.02.2012).

Decreto 26/2010, de 23 de febrero, del Gobierno de Aragón, por el que se modifican las Directrices Parciales de Ordenación Territorial del Pirineo Aragonés aprobadas por Decreto 291/2005, de 13 de diciembre, del Gobierno de Aragón (2010, 8 de marzo). En Boletín Oficial de Aragón, no 46 [en línea]. Disponible en: http://www.boa.aragon.es/cgi-bin/EBOA/BRSCGI?CMD=VEROBJ\&MLKOB=507924135959

Decreto 291/2005, de 13 de diciembre, del Gobierno de Aragón, por el que se aprueban las Directrices Parciales de Ordenación Territorial del Pirineo Aragonés (2005, 28 de diciembre). En Boletín Oficial de Aragón, no 153 [en línea]. Disponible en: http://www.boa.aragon.es/cgibin/EBOA/BRSCGI?CMD=VEROBJ\&MLKOB=97738980202

Decreto legislativo 1/2006, de 27 de diciembre, del Gobierno de Aragón, por el que se aprueba el texto refundido de la Ley de Comarcalización de Aragón (2006, 30 de diciembre). En Boletín Oficial de Aragón, no 149 [en línea]. Disponible en: http://www.boa.aragon.es/cgi$\mathrm{bin} / \mathrm{EBOA} / \mathrm{BRSCGI}$ ?CMD=VEROBJ\&MLKOB=167403580404

Ebrópolis, Asociación para el Desarrollo Estratégico de Zaragoza y su Entorno. Informe de Indicadores 2011: Estrategia Zaragoza 2020. Disponible en: http://www.ebropolis.es/web/index.asp

Ebrópolis, Asociación para el Desarrollo Estratégico de Zaragoza y su Entorno. Marco Estratégico Zaragoza 2020. Disponible en: http://www.ebropolis.es/web/index.asp

Gobierno de Aragón. Departamento de Política Territorial e Interior (2013) Estrategia de Ordenación Territorial de Aragón (EOTA). Disponible en: http://www.aragon.es/eota

Jenks, G.F. (1967) The data model concept in statistical mapping. International Yearbook of Cartography, 7, 186-190.

Levitt, T. (1981) El ciclo de vida del producto: gran oportunidad de marketing. Harvard Deusto Business Review, 6, 5-28.

Ley 4/2009, de 22 de junio, de Ordenación del Territorio de Aragón. En Boletín Oficial de Aragón, no 124 (en línea): http://www.boa.aragon.es/cgi-bin/EBOA/BRSCGI?CMD=VEROBJ\& MLKOB=384854643939 (Consulta: 06.02.2012).

Ley 7/1998, de 16 de julio, por la que se aprueban las Directrices de Ordenación Territorial para Aragón. En Boletín Oficial de Aragón, no 89 (en línea): http://www.boa.aragon.es/cgi-bin/ EBOA/BRSCGI?CMD=VEROBJ\&MLKOB=578882914544 (Consulta: 10.02.2012).

Martin, R., Sunsley, P. (2006) Path dependence and regional economic evolution. Journal of economic geography, 6(4), 395-437.

Mendez, R. (2010) Estrategias de innovación industrial y desarrollo económico en las ciudades intermedias de España, Fundación BBVA, $1 \stackrel{\text { a }}{\text { Ed. }}$

Observatorio de la Sostenibilidad en España (2010). Sostenibilidad en España 2010. (en línea): http://www.sostenibilidad-es.org/sites/default/files/_Informes/anuales/2010/ sostenibilidad_2010_0.pdf (Consulta: 06.03.2012). 
Polese, M. (2010) The resilient city on the determinants of successful urban economies. Montreal, Urbanisation, culture, societe, INRS (en línea): http://epe.lac-bac.gc.ca/100/200/300/inrsucs/inedit_working_paper/2010/n03/inedit2010_03.pdf (Consulta: 13.03.2012).

Prada, J. (2012) Estrategias, actores y redes en la revitalización de ciudades industriales en declive: el caso de Langreo (Asturias). Ciudad y Territorio. Estudios Territoriales, 174, 707-725.

Research Institute for Knowledge Sistems (s.f.) Metronamica (en línea): http://www.metronamica.nl/index.php (Consulta: 01.10.2012).

Rodríguez, E. J. (2013). Detroit: así se hundió el Titanic del capitalismo estadounidense. Jot Down Cultural Magazine (en línea): http://www.jotdown.es/2013/02/detroit-asi-se-hundio-eltitanic-del-capitalismo-estadounidense (Consulta: 04.03.2013).

Röhring, A., Gailing, L. (2010) Path Dependency and Resilience - The Example of Landscape Regions. German Annual of Spatial Research and Policy, 6, 79-88.

Sánchez, S., Prada, J., Méndez, R. (2009) Dinámicas de las ciudades de tamaño intermedio en el sistema urbano español: entre el declive y la recuperación. En Actas del XXI Congreso de Geógrafos Españoles (p. 655-670). Ciudad Real: Universidad de Castilla-La Macha, Departamento de Geografía.

Serrano, A. (2012) Modelo territorial y cambio global: el horizonte español a medio plazo. Ciudad y Territorio. Estudios Territoriales, 171, 11-36.

Slomp, H.J. (2004) La despoblación del medio rural español (Tesis de licenciatura en Geografía Humana). Universidad de Groningen, Facultad de ciencias Espaciales, Países Bajos.

Sorabji, R. (2004) The philosophy of commentators, 200-600 AD: Psychology (with ethics and religion). London: Duckworth.

Unión Europea. Agenda Territorial de la Unión Europea 2020: hacia una sociedad integradora, inteligente y sostenible para una Europa de Regiones diversas (en línea):

http://www.eu2011.hu/files/bveu/documents/TA2020.pdf (Consulta: 01.02.2012)

Unión Europea. ESPON. Inspire policy making by territorial evidence. ESPON HyperAtlas (en línea): http://www.espon.eu/main (Consulta: 01.10.2012). 
